\title{
Automatically Extract the Left Ventricular Myocardium by Using Active Contour Model
}

\author{
Jackulin DuraiRani. $A^{1}$, Sivaranjini.B ${ }^{2}$, Rekha. $A^{3}$ \\ PG Scholar, Dept., of Computer Science and Engineering, Dr.N.G.P. Institute of Technology, Tamil Nadu, India ${ }^{1,2,3}$
}

\begin{abstract}
This paper completely describes the fully automatic segmentation system for left ventricular myocardium segmentation from CT images by using active contour model. The system follows a coarse-to-fine strategy by first restricting the left ventricle and then damaging the myocardial surfaces of the left ventricle for segmentation. Particularly, the blood surface of a CT image is removed and illustrated as a triangular surface. Then, the ventricle is localized as a salient element on these surface victimization geometric and anatomical characteristics. After that, the heart muscle surfaces square measure initialized from the localization result and evolved by applying forces from the image intensities with a constraint supported the initial heart muscle surface locations.
\end{abstract}

Keywords: Active contour, ASM, PCA, AAM, Myocardium.

\section{INTRODUCTION}

A cardiovascular disease is the source for 17.5 million for myocardium segmentation. Active appearance models deaths in the world. The early diagnosis of the are expanding this idea by including gray scale level cardiovascular diseases plays an important role in the information and mostly used in segmenting the left and recovery of cardiovascular disease. Cardiac MRI imaging right ventricle myocardium from MR images. These using cine MRI, plays an important role that can be used ASMs and AAMs are restricted to the shape space where for examine cardiac function. Examination of cardiac the heart models are embedded. A more experienced way function needs calculation of different cardiac parameters to represent the shape by 3D diffusion wavelets. Using (i.e. ejection fraction (EF), left ventricle mass (LVM), left deformable models provides a versatile thanks to ventricle volume. All those parameters are depends on incorporate form priors that are capable of adapting to segmenting the endocardium, and epicardium contours of native image content. As an example, Ecabert et al. the left ventricle from the image sequences that are shapely the complete heart as a multi-compartment, acquired from cardiac imaging technique. The manual triangular surfaces.

segmentation takes a lot of time and effort from the The native adaptation was achieved by increasingly cardiologist for those contours. So that, the automatic optimizing the piecewise affine transformations of this segmentation is very needful, and that can be considered as a difficult task.

Early identification of myocardium dysfunction through quantitative analysis permits a reliable and fast diagnosis of heart diseases. Such quantitative functions include left ventricle ejection fraction, left ventricle myocardium thickening over the cardiac cycle and left ventricle myocardium mass. To evaluate these measures, accurate delineation of the left ventricle cavity and left ventricle cardiac muscle is required.

\section{RELATED WORKS}

The main difficulties in segmenting the myocardium is that large shape deviation within cardiac cycles and myocardium was different for between different patients, and weak edges between myocardium and heart tissues. For an precise and durable segmentation, model-based methods have become ascendant in segmentation. Heart models are mainly used for representing the geometric or potency features of the heart, and they can be used either explicitly or implicitly for segmenting heart surfaces. In the First methods, models are created in the off-line and those models are fitted for images in image segmentations. For example, active shape models that gives a statistical shape model for a set of affiliated shapes with the principal component analysis (PCA) technique, and used model to match image boundaries. In a mean form of the centre was fitted to a picture by estimating similarity transformations that was then distorted to match image boundaries with the assistance of landmark points on the interventricular septum. Rather than deforming a prealigned model, atlas-based ways use form information implicitly by directly registering each atlas image to a destination image.

Active contour models are mostly used in segmentation of medical images because of their stretchability, and robustness. In those models, energy functionalities are widely defined over features like edges, region statistics, local characteristics, and a combination of edges and regions of the medical images which are defined by gradient value techniques. Previous information can be absorbed well to limit the optimization space.

A various attributes of region-based active contours is that the initial contours can be located anywhere in the image as region-based segmentation relies on the global energy minimization rather than local energy minimization. Therefore, less prior knowledge is required than edge-based active contours. Traditional region-based active contours partition an image into multiple sub regions, those multiple regions belong to only two subsets: either the inside or the outside of contours. 
III.

\section{METHOD OVERVIEW}

This paper represents complete automatic system for segmenting the myocardium form CT images without trained images. The following flow chart [21] that represents a automatic segmentation of myocardium from CT images.

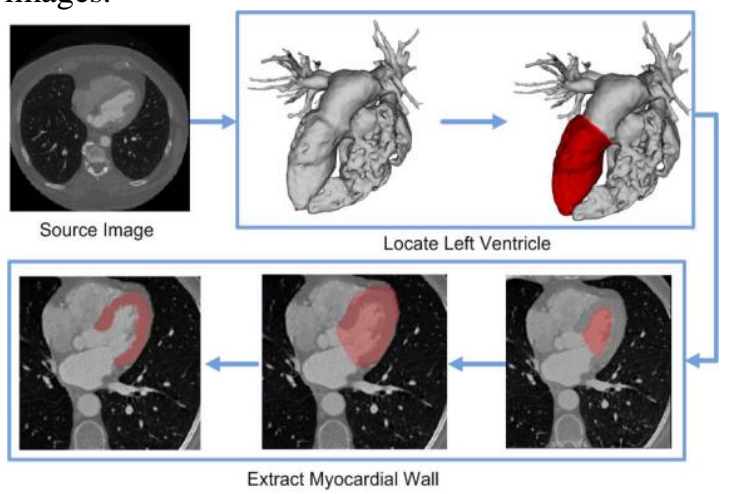

Fig 1.Method overview

Before starting the automation system step, the heart surface is estimated by the blood pool surface. Then, we detect the apex point of the left ventricle by using the relative physical coordinate of the system in CT images. The heart ventricle is mechanically detected by examining the distribution of the extent sets ranging from the apex purpose that is additional refined by activity the geometric active contour model on the blood pool surface. This contour decomposes the surface into 2 components, and also the one contains the apex purpose is chosen because the initial endocardium surface. Once the endcoardial surface is found, its corresponding mask is obtained via pasteurization. Then, a variational region-growing methodology is employed to extract the initial epicardial surface supported the saros segmentation. Finally, these 2 surfaces square measure refined by using a vigorous contour model with a form constraint, and also the heart muscle is obtained by extracting voxels between these surfaces. The following steps that can be involved in automatic segmentation of myocardium:

1. Left ventricle localization and myocardial wall segmentation

2. Endocardium Segmentation

3. Epicardium Segmentation

4. Myocardium wall segmentation

\section{A. Left ventricle localization and myocardial wall} segmentation

Left ventricular localization can be done by the following two ways

\section{Extract Blood Pool Surface \\ 2. Detect the Apex point}

\section{Extract Blood Surfaces}

Extraction of blood pool surface is done by the mature technique in computer graphics. The threshold value can be calculated to represent the heart image. And also morphological function can be applied to remove the noise and cut the spine in the component of heart from the CT image.

Detect the apex point

In this system, the XYZ correlates trace from left to right, rearward to anterior. The long axis of human being heart has unusual orientations, the directions of left and right is patently described from the inferior view. One of the salient features is apex point that is very useful to locate the left ventricle. Its location is described as follows: 1) estimate the correlation of ventricles; 2) Calculate the left ventricle apex point, which is the left tip point with respect to the estimated correlation.

To estimate the correlation point of left ventricles, the boundary of the blood surface Mbp is constructed first. Correlation of human blood pool surfaces in the source image coordinate system as shown in the Fig 2 [21]

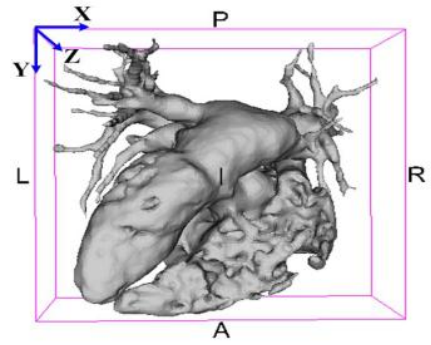

Fig 2. Correlation of human blood surface

The reference directions are left $(\mathrm{L})$, right $(\mathrm{R})$, posterior $(\mathrm{P})$, anterior (A), inferior (I), and superior (S). The vertices used for calculating the ventricle correlation are defined as follows

$\mathrm{V}_{\mathrm{ch}}(\mathrm{p} \square)=\left\{\mathrm{p} \square \mid \mathrm{k}(\mathrm{P} \square) \mu_{\mathrm{K}}+\sigma_{\mathrm{K}} \cap \mathrm{Y}\left(\mathrm{p}_{\square}>\mathrm{tv}\right\}\right.$

Where $\mu K$ are mean and $\sigma K$ are the standard deviation of $K(\sim p)$, and a threshold value $t y$ that defines the region of interest for the left ventricles, which was calculated as follows

$$
t y=y \min +0.5(y \max -y \min ) .
$$

The PCA technique is very useful to find the principal component of these translated points ps as the orientation of the ventricles, denoted by $\mathbf{H}$. The positive direction is chosen so that $\mathbf{H}$ has negative component in the $\mathrm{Y}$ direction. A plane $L O$ passing through $\mu \mathrm{bp}$ with normal $\mathbf{N}$ $=\mathbf{Z} \times \mathbf{H}$ defines a reference plane such that the left ventricle points are mainly above the plane and otherwise for the right ventricle.

\section{B. Endocardium Segmentation}

The serosa is that the innermost layer of heart that lines the chambers of the centre. Its cells area unit embryologic ally and biologically similar to the epithelial tissue cells that line blood vessels. The serosa conjointly provides protection to the valves and heart chambers.

The serosa underlies the way more voluminous heart muscle, the muscle liable for the contraction of the centre. The outer layer of the centre is termed visceral and therefore the heart is encircled by a little quantity of fluid embedded by a fibrous sac known as the pericardium. 
The segmentation of the serosa depends on segmenting the 55 cavity, by extracting completely different options (i.e. the gradient magnitude, the most important Eigen value, the output of median filter, and therefore the grey value) from every element within the image.

We tend to selected the options (used within the phaseation) attributable to the character of the 55 cavity that we wish to segment it. The 55 cavity seems bright and therefore the heart muscle that surrounds it seems dark. So, we want to boost edges that surround the 55 cavity.

\section{Epicardium Segmentation}

The serous membrane may be a double-walled sac containing the centre and therefore the roots of the good vessels. The sac has 2 layers, a humour layer and a fibrous layer. It encloses the cavum that contains serosa fluid.

The serous membrane fixes the centre to the cavum, offers protection against infection, and provides the lubrication for the centre.

There are unit completely different tissues that surround the heart muscle, and these tissues have completely different intensity values. Also, the part of the visceral pericardium before of the correct ventricle is skinny in thickness. All of those problems create the segmentation of the visceral pericardium is tough.

\section{Myocardium wall segmentation}

The myocardial is described as the volume between the endocardium and epicardium masks. Note that the contour evolution process returns closed masks. To segment a complete myocardium wall, the blood pool surface that has to be removed which is residing inside the voxel. To this end, the wall is segmented into two parts: one that contains the myocardium that can be completely calculated by the XOR operation between the endocardium and epicardium masks, and the one formed by removing the voxels within the blood pool surface from the epicardium mask.

\section{DISCUSSION AND FUTURE WORK}

We have introduced a complete automatic system for myocardium wall segmentation from CT images. It follows the coarse-to-fine strategy by first restricting the left ventricle and then damaging the myocardial surfaces of the left ventricle for segmentation and then refining this result by clarifying contour evolution techniques with a shape constraint obtained on-line. The performances have been evaluated by calculating the errors between automatic and manual segmentations. The proposed method was very specific for the left ventricular myocardium wall segmentation, it may be utilized in several possible ways for broader applications in cardiac image segmentation

In future work, we planned to segment left and right ventricles in CT images and apply Region based segmentation results to clinical applications such as evaluating the myocardial mass at risk caused by stenos.

\section{REFERENCES}

[1]. Liangjia Zhu, Yi Gao, Vikram Appia, Anthony Yezzi, Chesnal Arepalli, Tracy Faber,Arthur Stillman, and Allen Tannenbaum, Fellow, IEEE "A Complete System for Automatic Extraction of Left Ventricular Myocardium From CT Images Using Shape Segmentation and Contour Evolution"

[2]. C. Petitjean and J. N. Dacher, "A review of segmentation methods in short axis cardiac MR images,” Med. Image Anal., vol. 15, no. 2, pp. 169-184, 2011.

[3]. T. F. Cootes, C. J. Taylor, D. H. Cooper, and J. Graham, “Active shape models-their training and application," Comput. Vis. Image Understand., vol. 61, no. 1, pp. 38-59, 1995.

[4]. H. C. van Assen, M. G. Danilouchkine, M. S. Dirksen, J. H. Reiber, and B. P. Lelieveldt, "A 3-D active shape model driven by fuzzy inference: Application to cardiac CT and MR," IEEE Trans. Inf. Technol. Biomed., vol. 12, no. 5, pp. 595-605, Sep. 2008.

[5]. H. C. van Assen, M. G. Danilouchkine, F. F. Frangi, S. Ordas, J. J. Westenberg, J. H. Reiber, et al., "SPASM: A 3D-ASM for segmentation of sparse and arbitrarily oriented cardiac MRI data," Med. Image Anal., vol. 10, no. 2, pp. 286-303, 2006.

[6]. T. F. Cootes, G. J. Edwards, and C. J. Taylor, "Active appearance models,” IEEE Trans. Pattern Anal. Mach. Intell., vol. 23, no. 6, pp. 681-685, Jun. 2001

[7 ]. S. C. Mitchell, B. Lelieveldt, R. J. van der Geest, J. G. Bosch, J. H. C. Reiber, and M. Sonka, "Multistage hybrid active appearance model matching: Segmentation of left and right ventricles in cardiac MR images," IEEE Trans. Med. Imag., vol. 20, no. 5, pp. 415-423,May 2001.

[8 ]. S. Essafi, G. Langs, and N. Paragios, "Hierarchical 3D diffusion wavelet shape priors," in Proc. Int. Conf. Comput. Vis., 2009, pp. 1717-1724.

[9]. O. Ecabert, J. Peters, H. Schramm, C. Lorenz, J. von Berg, and M. Walker, "Automatic model-based segmentation of the heart in CT images,” IEEE Trans. Med. Imag., vol. 27, no. 9, pp. 1189-1201, Sep. 2008.

[10]. X. Zhuang, K. S. Rhode, R. S. Razavi, D. J. Hawkes, and S. Ourselin, "A registration-based propagation framework for automatic whole heart segmentation of cardiac MRI," IEEE Trans. Med. Imag., vol. 29, no. 9, pp. 1612-1625, Sep. 2010.

[11]. V. S. Lempitsky, M. Verhoek, J. A. Noble, and A. Blake, "Random forest classification for automatic delineation of myocardium in realtime 3D echocardiography," in Proc. 5th Int. Conf. Funct. Imag. Model. Heart, 2009, pp. 447-456.

[12]. S. Kichenassamy, A. Kumar, P. Olver, A. Tannenbaum, and A. Yezzi, "Gradient flows and geometric active contour models," in Proc. 5th Int. Conf. Comput. Vis., Washington, DC, USA, 1995. pp. 810-815.

[13]. V. Caselles, R. Kimmel, and G. Sapiro, "Geodesic active contours,” Int. J. Comput. Vis., vol. 22, no. 1, pp. 61-79, 1997. [17] T. F. Chan and L. A. Vese, "Active contours without edges," IEEE Trans. Image Process., vol. 10, no. 2, pp. 2029-2039, Feb. 2001.

[14]. X. Wang, D. S. Huang, and H. Xu, “An efficient local Chan-Vese model for image segmentation,” Pattern Recognit., vol. 43, no. 3, pp. 603-618, 2010.

[15]. L. Chen, Y. Zhou, Y. Wang, and J. Yang, "Rapid and brief communication: GACV: Geodesic-aided C-V method," Pattern Recognit., vol. 39, no. 7, pp. 1391-1395, Jul. 2006.

[16]. S. Lankton, D. Nain, A. Yezzi, and A. Tannenbaum, "Hybrid geodesic region-based curve evolutions for image segmentation," Proc. SPIE, vol. 6510, p. 65104U, Mar. 2007.

[17]. S. Lankton and A. Tannenbaum, "Localizing region-based active contours," IEEE Trans. Image Process., vol. 17, no. 11, pp. 20292039, Nov. 2008.

[18]. A. Tsai, A. Yezzi, W. Wells, C. Tempany, D. Tucker, A. Fan, et al., "A shape-based approach to the segmentation of medical imagery using level sets," IEEE Trans. Med. Imag., vol. 22, no. 2, pp. 137-154, Feb. 2003.

[19]. V. Appia, B. Ganapathy, A. Abufadel, A. Yezzi, and T. Faber, "A regions of confidence based approach to enhance segmentation with shape priors," Proc SPIE., vol. 7533, p. 753302, May 2010.

[20]. V. Appia, B. Ganapathy, A. Yezzi, and T. Faber, "Localized principal component analysis based curve evolution: A divide and conquer approach," in Proc. IEEE Int. Comput. Vis. Conf., Nov. 2011, pp. 1981-1986 